\title{
Study of the influence of radius and distance between windings of Helmholtz coils for detection of cracks using active thermography in coil link chains.
}

\author{
by Pablo López de Uralde*, Eider Gorostegui-Colinas*, Iñaki Silanes*, Beñat Urtasun*, Rafael \\ Hidalgo*, Iratxo Gómez ${ }^{\dagger}$ and Iñaki Fdz de Bastida ${ }^{\dagger}$.
}

*LORTEK Technological Centre, Basque Research and Technology Alliance (BRTA), Arranomendia kalea, 4A, Ordizia, 20240, Spain

† CS Centro Stirling, Araba hiribidea, 3, 20550, Aretxabaleta, Gipuzkoa, Spain

\begin{abstract}
The detection of surface defects in coil link chains is essential to ensure their correct operation in adverse conditions, such as offshore applications. During the processes of bending and welding of the links, cracks that lead to structural failure under working conditions can be generated. That is why an early detection is necessary, especially for safety, but also for economic reasons.
\end{abstract}

Traditional Non Destructive Testing methods in industry, such as Dye Penetrant Testing or Magnetic Particles, are in general slow and need an experienced technician both in conducting the tests and in interpreting the results. Furthermore, those methods imply the need of cleaning the test specimen after finishing, increasing times and costs. Consequently, inductive thermography has been identified as an advantageous technique because of its cleanness, fastness and the possibility of automation of the whole inspection procedure [1] [2] [3].

The aim of this work is the optimization of the inspection of offshore chains via inductive thermography. For this reason, a research between different types of coils has been performed, leading to the conclusion that Helmholtz coils fit with the needs of the industrial inspection. Prior to the detection and location of cracks, a complete study of the influence of two parameters has been carried out: coil radius and distance between windings. These parameters are essential for the inspection of chains during production, due to the reduced dimensions and complicated accessibility on industrial environment.

In order to study the influence of these two parameters, an analytical model has been developed and the deduced conclusions have been corroborated by means of experimental measurements with different size coils. Finally, the optimal range of parameters has been extracted and applied for the detection of cracks on a welding part of a link.

\section{Introduction}

Recently, the increase in the demand for automation of both production and inspection processes of companies and the interest in data extraction that increase the knowledge of the production process, have led inspection techniques to adapt and respond to these demands. That is why different sectors are requiring a change from traditional Non Destructive Testing (NDT) to advanced inspection techniques. The final objective is to implement in the factory an automatic, reliable, fast inspection system that does not depend on subjective criteria.

In particular, Lortek has been working for almost 5 years on the substitution of Magnetic Particles by Inductive Thermography in the inspection of offshore chains (Fig. 1) for the detection of superficial defects. In order to respond to this need, a study on the viability of inductive thermography as a solution has been necessary, facing the double challenge of creating a system capable of (i) detecting all the defectology nowadays covered by magnetic particles automatically and (ii) solving the accessibility problems arising due to the harsh environment in which chains are manufactured.

Crack appearance during the manufacturing of coil links can be due to different reasons, such as inherent defects in the employed material, a defective welding process or ineffective grip of the link during the welding process and cooling. Each of them can lead to cracks in different areas and with a different typology. For example, previous delamination in the raw material generates superficial, prolonged and longitudinal cracks; welding issues can provoke cracks in the welded area itself, as well as the grip jaws can crack the link in the elbow areas.

For these reasons, the inspection of the most of the surface of the link is necessary. Furthermore, in order to avoid stopping the production, or making it slower, it is mandatory to have a fast inspection system, which in this case, due to the production design in the factory, means to inspect a chain link faster than it is produced. To solve this challenge, a solution that allows the inspection of large areas and so that accessibility is as complete as possible must be proposed. 


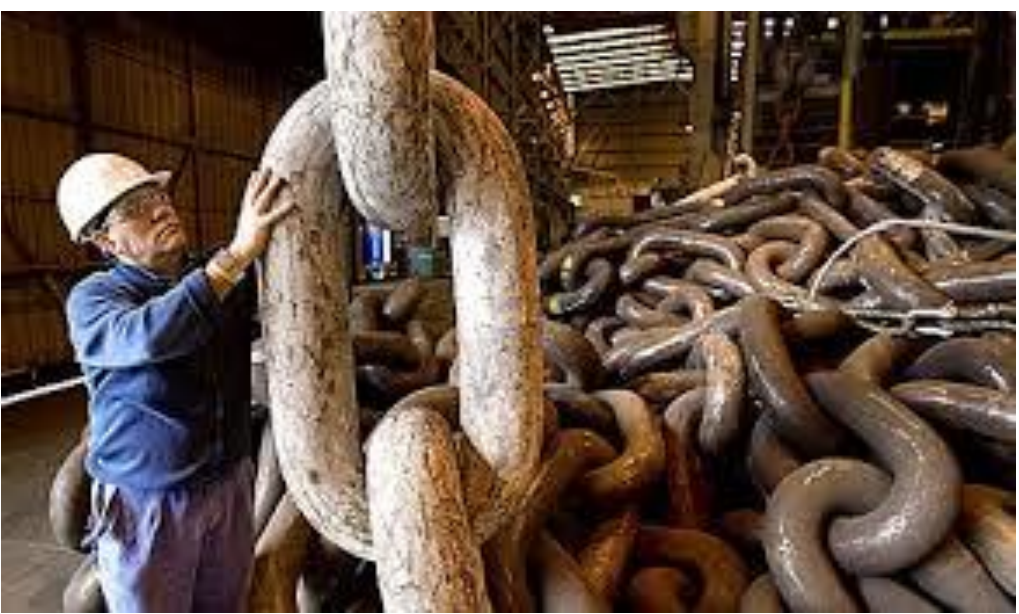

Fig. 1. Large coil link chains (Source: VICINAY Marine).

Among the different inductor geometries analysed, there are Helmholtz type coils. This type of coil, consisting of a double circular winding, generates a constant magnetic field between both circular sections when the distance between them is equal to the radius (Fig. 3). Due to its geometry, it is applicable to situations where samples with a cylindrical shape must be inspected [4]. For this reason, an analytical and experimental study has been carried out to optimize the design of these coils for using in the detection of cracks by inductive thermography on steel links.

\section{Inductive thermography}

Several excitation techniques are employed in active thermography for NDT crack detection [5]. Those excitations can be optical (laser or lamp based), ultrasonic waves or electric current. The basics for all of them are similar: the sample to be inspected is subjected to an external excitation and a thermal camera captures the heat propagation along the sample. This propagation depends on the thermal properties of the material, and the existence of anomalies lead to differences in this distribution that derive in temperature differences in the surface [6].

Induction heating is based on the generation of magnetic losses in the material to be heated. The primary mechanism of heat generation by this technique is due to the well-known induced Eddy currents. According to Faraday's law, when a conductive material (material to be heated) is crossed by a magnetic field, electromotive forces appear in it causing the circulation of eddy currents called Eddy currents.

In general, induction heating offers the following advantages:

- High productivity: the heat is generated directly and instantaneously in the piece to be analysed.

- Control and automation of the process.

- Rapid heating: the piece is heated directly, which drastically reduces the thermal inertias of the heating process.

- Green energy: induction is a clean and non-polluting process.

- Security: since no flame is generated.

The effectiveness of the induction heating process depends on several factors:

- Material of the piece to be heated: the material must be electrically conductive and/or have a wide hysteresis cycle, which is why metals are normally used.

- Electromagnetic properties: materials with high electrical resistivity accumulate heat more easily. Higher relative magnetic permeability causes more superficial heating in the sample.

- Inductor design: well-designed inductor takes into account the proper pattern for heating an area and maximizes generator efficiency. The constructive aspects of the inductor are essential to maximize the amount of heat for the same generator power

- Induction generator capacity: the higher the generator power, the higher temperatures can be reached. Greater power also allows the range of possible inductors to be used to be expanded. As far as the induction frequency of the equipment is concerned, the high frequencies cause the generation of eddy currents in the most superficial layers of the piece to be analysed, so that the heating will be superficial. In contrast, lower frequencies induce currents at greater depths, being able to heat beyond the surface of the piece. This occurs due to the so-called "skin" effect, which causes the current to move closer to the external surface of the conductor the higher the frequency. 
Generally, the defects detected by this technique are superficial or very close to the surface, since Devices Under Test (DUT) are habitually composed by metallic materials, the heat disperses quickly and deep depths cannot be reached. An important concept in inductive thermography tests is skin depth or electromagnetic penetration, that is, the depth at which induced Eddy currents reach. The skin depth is defined according Equation 1:

$$
\delta=\sqrt{\frac{\rho}{\pi \mu_{\mathrm{r}} \mu_{0} \mathrm{f}}}
$$

Where $\rho, f, \mu 0$ and $\mu \mathrm{r}$ represent respectively the electrical resistivity [ $\Omega . m]$, the induction frequency [Hz], the magnetic permeability constant $[\mathrm{H} / \mathrm{m}]$ and the relative magnetic permeability of the material.

Therefore, it can be deduced that, for a greater detection capacity of surface defects, the skin depth has to be as low as possible. On the contrary, to detect subsurface defects, it must have a higher value (Fig 2).
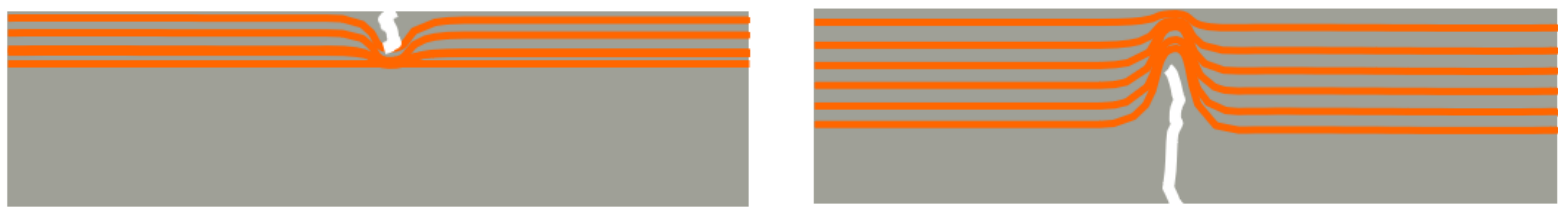

Fig 2. Low skin depth for surface defectology (left), high skin depth for sub subsurface defectology (right).

\section{An Analytical Model}

Inductive thermography NDT is based on heating the DUT by means of magnetic losses. These losses can be computed employing the loss separation method (Equation 2) introduced by Bertotti in [7], which is based on the Steinmetz formulation [8], [9].

$$
P_{\text {mag }}=P_{\text {hyst }}+P_{\text {class }}+P_{\text {exc }}=K_{h} \cdot B_{m}^{2} \cdot f+\frac{\pi^{2} \cdot \sigma \cdot a^{2}}{6} \cdot(B \cdot f)^{2}+K_{\text {exc }} \cdot(B \cdot f)^{3 / 2}
$$

Where $P_{\text {hyst }}$ represents hysteresis power losses and the two components of the dynamic losses are created by induced Eddy Currents, $P_{\text {class, }}$ and excess losses, $P_{\text {exc. }}$

In any case, Joule heating or losses related to Eddy currents are sole mechanism of heat generation in nonmagnetic materials and the primary mechanism in ferromagnetic [10]. Consequently, Equation 2 can be simplified into Equation 3:

$$
P_{\text {mag }}=P_{\text {class }}=\frac{\pi^{2} \cdot \sigma \cdot a^{2}}{6} \cdot(B \cdot f)^{2}
$$

Where $\sigma$ is the electric conductivity of the material $[\sigma / \mathrm{m}]$ and $a$ is the thickness of the DUT [m]. Therefore, magnetic flux density, $B[\mathrm{~T}]$, and frequency, $f[\mathrm{~Hz}]$, are the variables in which we should be focused on to improve the heating process. Knowing that the frequency is defined by the power electronics, the improvement of the heating system by means of the inductors is related to the magnetic flux density that cross the DUT.

In this study, Helmholtz coils have been selected as a feasible option for the inspection of offshore chains. The analysed design parameters of these coils that have influence in the created magnetic flux density are shown in Fig. 3: coil radius, $R_{a}$, and distance between windings, $l_{e}$. 


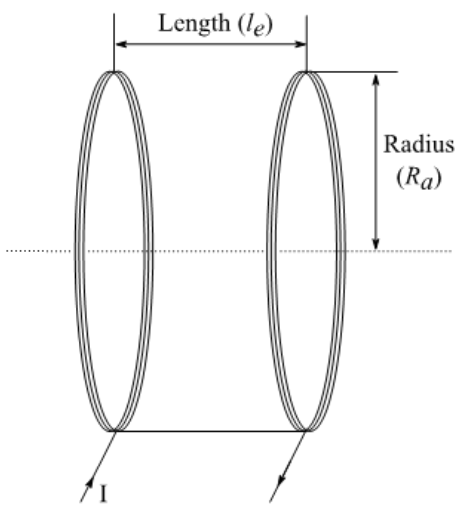

Fig. 3. Design parameters of a Helmholtz Coil. 4:

Applying the well-known magnetic relations, the magnetic flux created by the coils could be computed by Equation

$$
\begin{aligned}
& \phi=B \cdot S \\
& B=\mu \cdot H \\
& H \cdot l_{e}=N \cdot i \\
& \phi=\frac{\mu \cdot N \cdot i \cdot \pi \cdot R_{a}^{2}}{l e}
\end{aligned}
$$

If the magnetic permeability, $\mu$, the number of turns, $N$, and the feed current, $i$, are constant, from (Equation 4 ) it can be deduced that an increase in the distance between windings, will decrease the flux and therefore the heating capacity. On the other hand, an increase in the coil radius, will increase both the magnetic flux and the heating capacity.

It is important to notice that Equation 4 can be employed to compute the magnetic flux created by the coils. However, this is not the magnetic flux that crosses the DUT. In order to obtain the magnetic flux that crosses the DUT, Equation 4 should be multiplied by a coupling factor between coils and DUT. This coupling factor is reduced with the increase of the distance between current conductors and DUT. In Fig. 4 can be observed the magnetic flux density in the DUT when it has a constant radius of $10 \mathrm{~mm}$ and the coil radius is modified. Therefore, it can be concluded that to increase the heating performance a good balance between the created magnetic flux and the coupling factor must be necessary. Normally, these performances of the heating systems are maximized when the coil is as close as possible to the DUT, touching it.

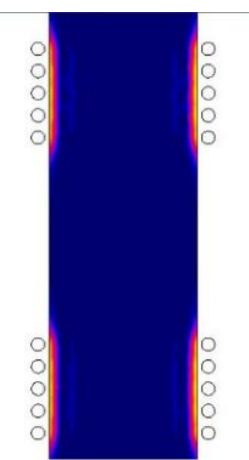

a) $R_{a}=11.5 \mathrm{~mm}$

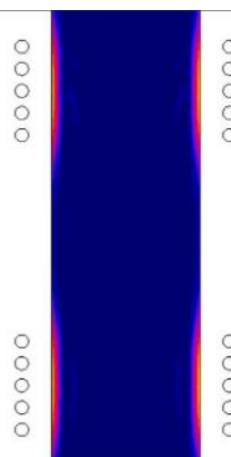

b) $R_{a}=14 \mathrm{~mm}$

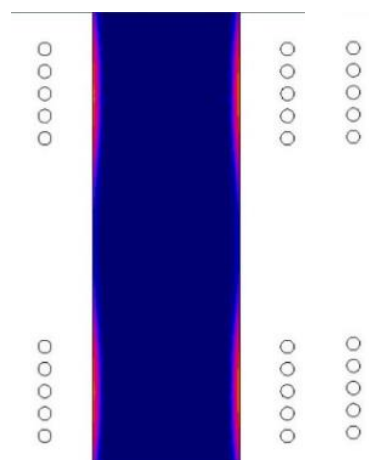

c) $R_{a}=16.5 \mathrm{~mm}$
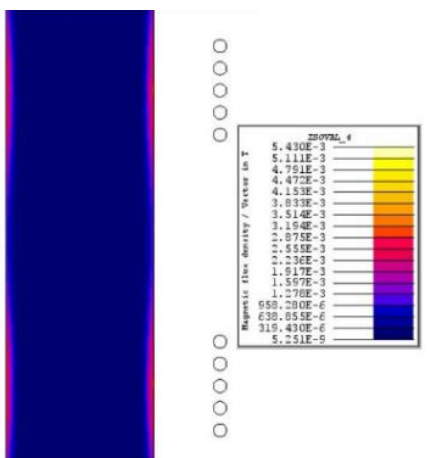

d) $R_{a}=19 \mathrm{~mm}$

Fig. 4. Magnetic flux density in the DUT. 


\section{Description of Experimental measurements}

Once stablished that Helmholtz coils fit the needs of the inspection, different challenges arisen from the selection have to be solved. Geometrically, the two main problems to avoid are how to open/close the coil in order to embrace the links and the calculation of winding dimensions to avoid collisions with the chain. Solving the first issue is out of the purpose of this paper, which focuses on the second one. The results of the analytical model show that the higher magnetic flux through the sample is achieved when the coupling factor is as high as possible, that is to say, with the wire directly on the link (Fig. 5).

However, this solution is not feasible from an industrial point of view for several reasons: wear of the wire due to friction with the link, variations of the chain link diameter with respect to the theoretical one due to manufacturing tolerances could generate areas where the wire can get stuck, etc. Thus, some tests with coil radius bigger than sample radius have been carried out. In these tests, the obtained heating has been greater than in tests where the inductor wire was in contact with the chain link. Taking into account the large size and high density of the real samples in an industrial environment, maximizing and optimizing the heating process have been declared a priority. Hence, after detecting these differences between the analytical model and the results obtained in the first experimental tests it has been decided to carry out the experimental study that gives rise to this article. The main objective of this work is to define the optimal radius of the coil and the distance between its windings to maximize the heating of the link using inductive heating.

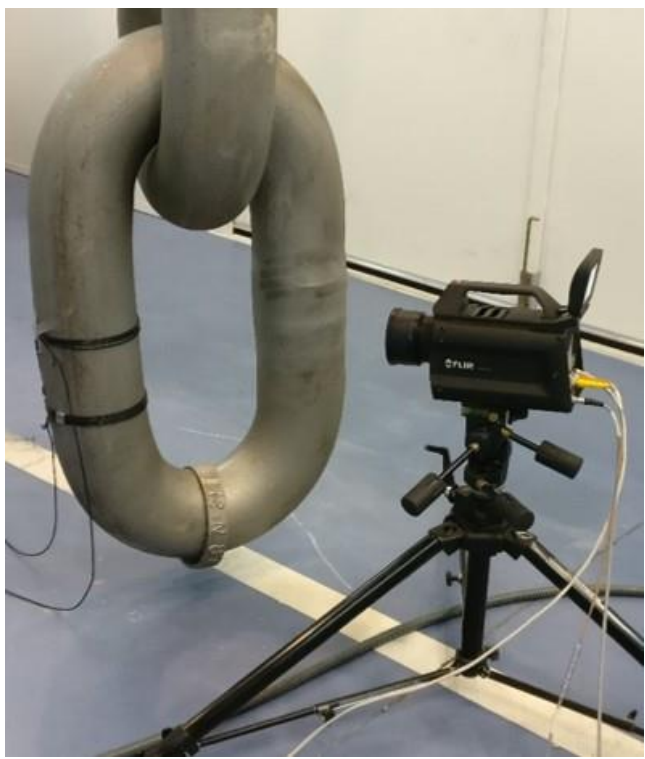

Fig. 5. Set up with wire mounted directly to the link.

For the experiments carried out in this study, three samples of different radius and materials have been selected (Fig 6). The purpose of choosing different materials has been to check if great differences could be detected in the results between different types of steel. In turn, the variation in radius of the samples is necessary to conclude the influence of this parameter on the results.

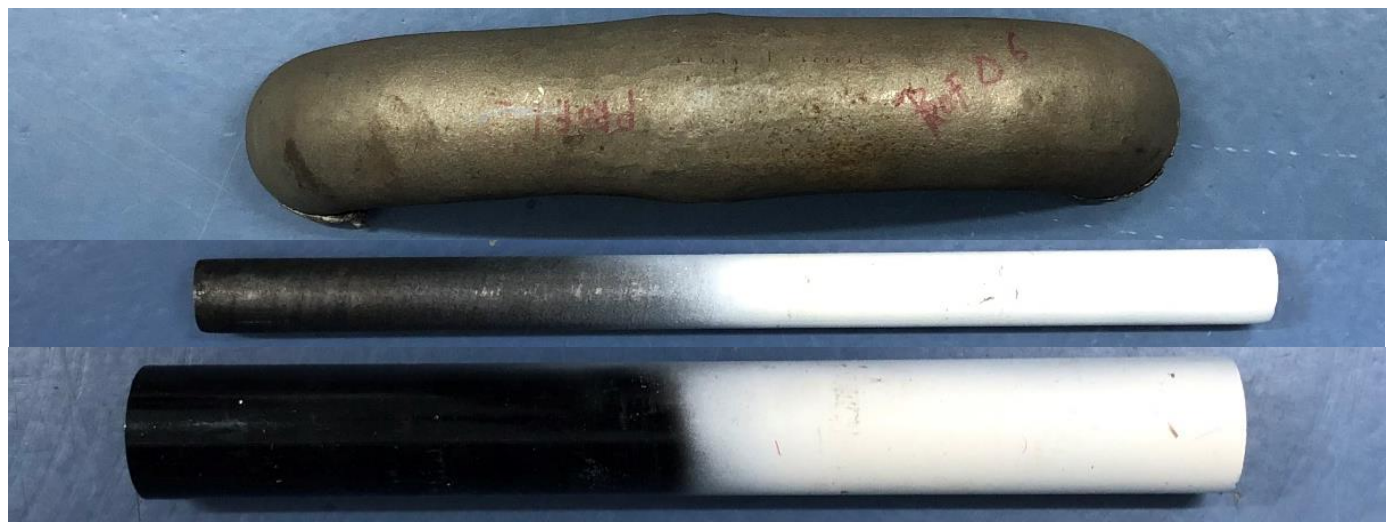

Fig 6. Sample 1 (top), Sample 2 (medium), Sample 3 (bottom). 
The first sample (Sample 1, from now on) is a piece of a real link, $40 \mathrm{~mm}$ radius. The material is steel quality $\mathrm{R} 3$. The second sample (Sample 2) is a carbon steel bar, $17 \mathrm{~mm}$ radius. Finally, an inox steel bar, $25 \mathrm{~mm}$ radius (Sample 3 ) (Table 1). This inox steel bar has been employed to check if using a non-ferromagnetic material could lead to different results compared with the others. Both Sample 2 and Sample 3 have been painted white to homogenise and increase the emissivity (even though this is not contemplated for the industrial inspection system). Sample 1 has remained as it would be in real inspection conditions, shot blasted.

Table 1. Samples characteristics.

$\begin{array}{cccc}\text { Sample } & \text { Material } & \text { Radius } & \text { Painted } \\ \text { Sample 1 } & \text { Steel Quality R3 } & 40 \mathrm{~mm} & \text { NO } \\ \text { Sample 2 } & \text { Carbon steel } & 17 \mathrm{~mm} & \text { YES } \\ \text { Sample 3 } & \text { Inox steel } & 25 \mathrm{~mm} & \text { YES }\end{array}$

The equipment for the inspections has consisted in:

- $\quad 3 \mathrm{~kW}$ Induction Generator, ITVis3000 (Edevis Gmbh). This generator allows excitation by short pulses of less than one second, with frequencies between 10 and $60 \mathrm{kHz}$.

- A Flir Infrared Cooled Thermal Camera has been used to perform the recordings, specifically a Flir $\mathrm{x} 6541 \mathrm{sc}$ with the standard $50 \mathrm{~mm}$ optics. This camera has a focal plane array InSb detector with a resolution up to $640 \times 512$ pixels, working in the short wave region at 1.5-5.5 $\mu \mathrm{m}$ and a Noise Equivalent Temperature Difference (NETD) of less than $25 \mathrm{mK}$ (typically $18 \mathrm{mK}$ ). The framerate has been set to $200 \mathrm{~Hz}$ for all measurements. Finally, integration time has been set to $1000 \mu \mathrm{s}$.

- $\quad$ Signal synchronization between camera and generator using ESG (Edevis Gmbh)

- Helmholtz coils with different radiuses and distance between windings.

Regarding the set up (Fig. 7), it has been reduced to the minimum needed. The camera has been placed at a fixed distance of $55 \mathrm{~cm}$, placing the coils at predetermined distances $(30-80 \mathrm{~mm})$ maintaining the sample centred inside the windings. For every configuration (sample / coil radius / distance between windings), reference induction parameters have been set:

- Induction Frequency between 20 y $40 \mathrm{kHz}$

- Pulse length between 100 y $300 \mathrm{~ms}$

- Power percentages between 40 y $100 \%$

These parameters have been combined depending on the sample to inspect, to obtain results with different configurations that could lead to better conclusions.

Finally, a $5 \mathrm{~kW}$ induction generator has been used (Edevis Gmbh) with Sample 1. These measurements have been stablished because the coil link chains diameter can increase to $220 \mathrm{~mm}$, so higher power could be needed to be able to have enough heating on them to detect possible cracks. The objective is to compare the results with the obtained using $3 \mathrm{~kW}$ and check if the conclusions could be extrapolated.

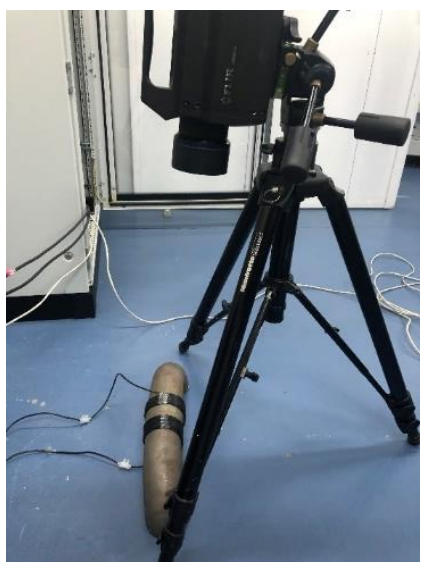

Fig. 7. Set up for Sample 1. 


\section{Experimental results}

Sample 1 has been chosen as an example to show how the results have been obtained and represented. Firstly, several tests have been carried out, modifying both induction parameters and geometrical parameters of the coils (Table 2).

Table 2. Ranges of induction and geometrical parameters employed.

$\begin{array}{ccc}\text { Frequency } & \text { Induction parameters } \\ \text { Pulse length } & 20-40 \mathrm{kHz} \\ \text { Power percentage } & & 100-300 \mathrm{~ms} \\ & \text { Geometrical parameters } & 40-100 \% \\ \text { Coil radius }\left(R_{a}\right) & & 17-65 \mathrm{~mm} \\ \text { Distance between windings }\left(l_{e}\right) & 30-80 \mathrm{~mm}\end{array}$

Once finished the tests, the measurements have been analysed. To do so, a rectangular Region Of Interest (ROI) in the centre zone between windings has been created (Fig. 8). Then, the time response of the average value of the ROI has been plotted, and the increase of the heating has been calculated. The heating has been expressed as Digital Levels (Intensity (DL)). These values have been organized in different tables, one for each combination of induction parameters (Table 3).

After that, two type of graphs have been generated: In the first one the radius of the coil (Ra) is fixed and the distance between the windings (le) is varied; in the second one the distance between windings (le) is fixed and the radius of the coil (Ra) is varied. In this way, the influence of each of the parameters has been analysed (Table 4).

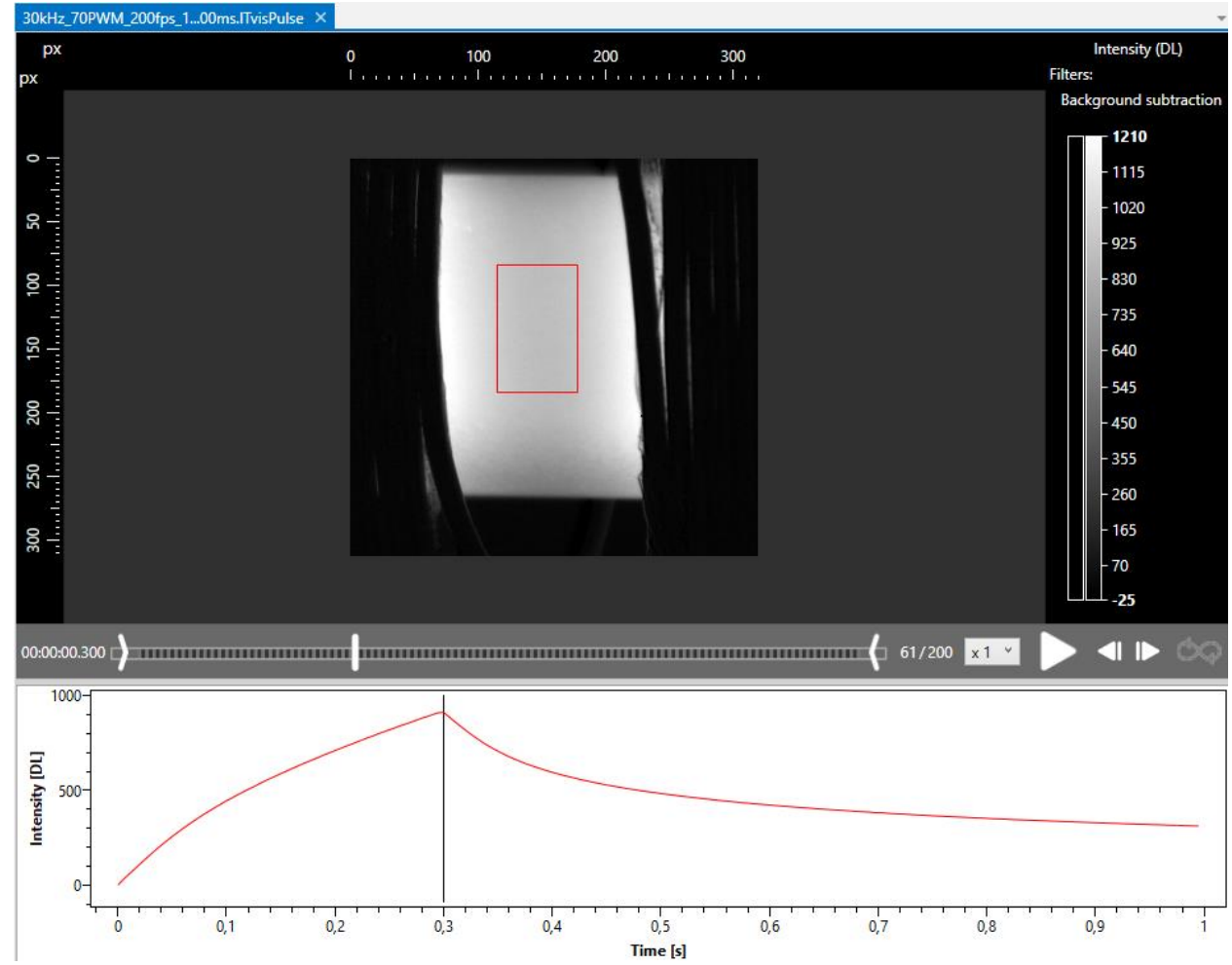

Fig. 8. ROI and time response of the average. 
Table 3. Data obtained from Sample 1 (30 kHz; 60\%; 300ms)

\begin{tabular}{|c|c|c|c|c|c|c|c|}
\hline \multicolumn{2}{|c|}{$30 \mathrm{kHz} ; 60 \% ; 300 \mathrm{~ms}$} & \multicolumn{6}{|c|}{ Distance between windings, le (mm) } \\
\hline & & 30 & 40 & 50 & 60 & 70 & 80 \\
\hline \multirow{4}{*}{$\begin{array}{c}\text { Coil radius, } \\
\mathrm{Ra}(\mathrm{mm})\end{array}$} & 40 & 139,019 & 90,986 & 53,757 & 36,53 & 22,413 & 16,947 \\
\hline & 45 & 189,219 & 124,278 & 83,391 & 54,816 & 40,921 & 30,058 \\
\hline & 55 & 142,828 & 97,4 & 66,846 & 43,449 & 33,179 & 23,974 \\
\hline & 65 & 93,527 & 72,049 & 48,21 & 34,909 & 23,459 & 17,115 \\
\hline
\end{tabular}

As shown in Table 4, the left side graph, where the distance between windings varies, does not give relevant information, as it was known that moving the coils away reduces the heating. However, in the right-side graph, can be seen that an optimum radius, higher than sample radius, exists.

Table 4. $\Delta$ Intensity vs Distance between windings (left) and $\Delta$ Intensity vs Coil radius (right) for Sample 1 (30 $\mathrm{kHz} ; 60 \% ; 300 \mathrm{~ms})$
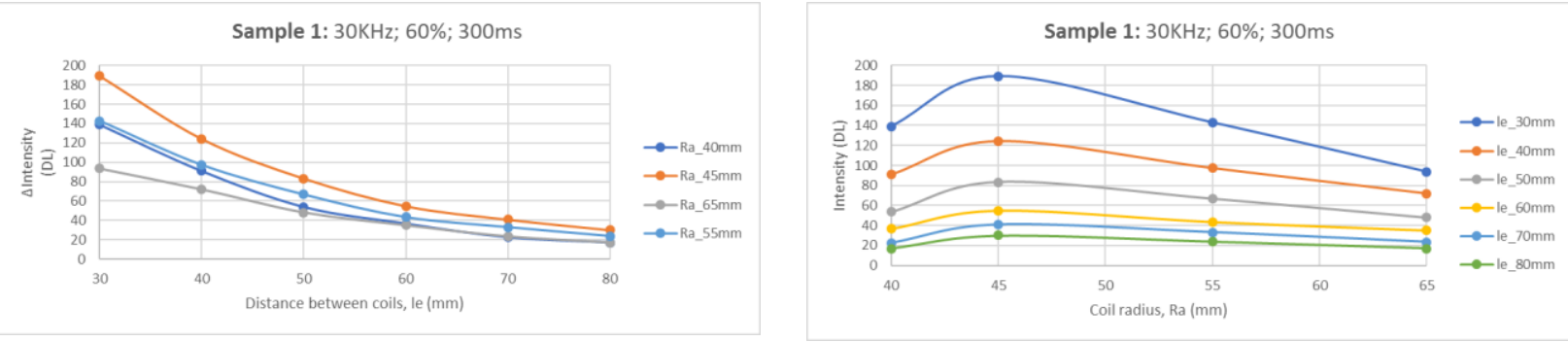

In order to establish a real comparison, the curves of interest ( $\Delta$ Intensity vs. coil radius) have been normalized with respect to their own mean and standard deviation. Finally, with the objective of reducing data to one graph and have a wider vision, the average of those curves at every point has been calculated. The curve obtained for the set of induction parameters of the example has been plotted in one graph (Table 5).

Table 5. $\Delta$ Intensity vs Coil radius raw data (top left), normalized (top right) and simplified (bottom) for Sample 1 (30 kHz; 60\%; 300ms)
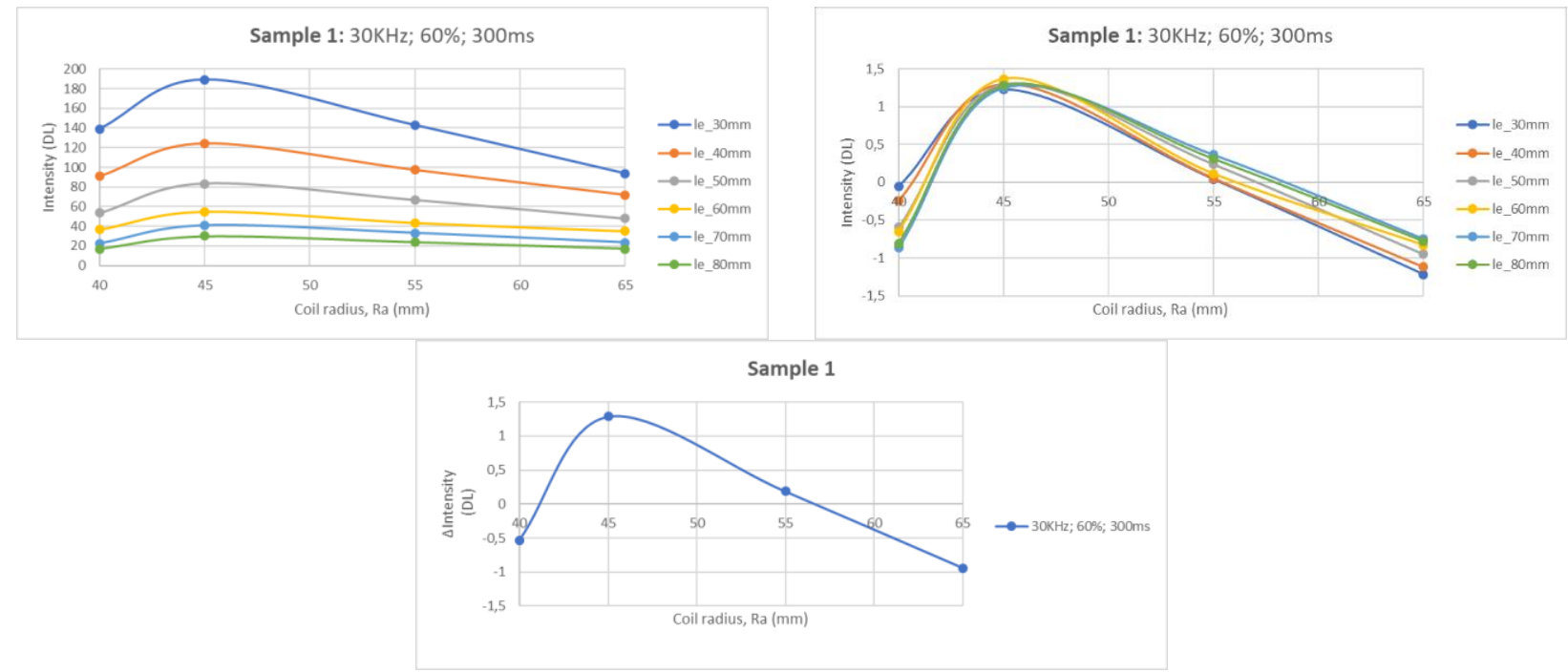
Table 6. $\Delta$ Intensity vs Coil radius, simplified data for every set of induction parameters. Sample 1 (top left), Sample 2 (top right) and Sample 3 (bottom).
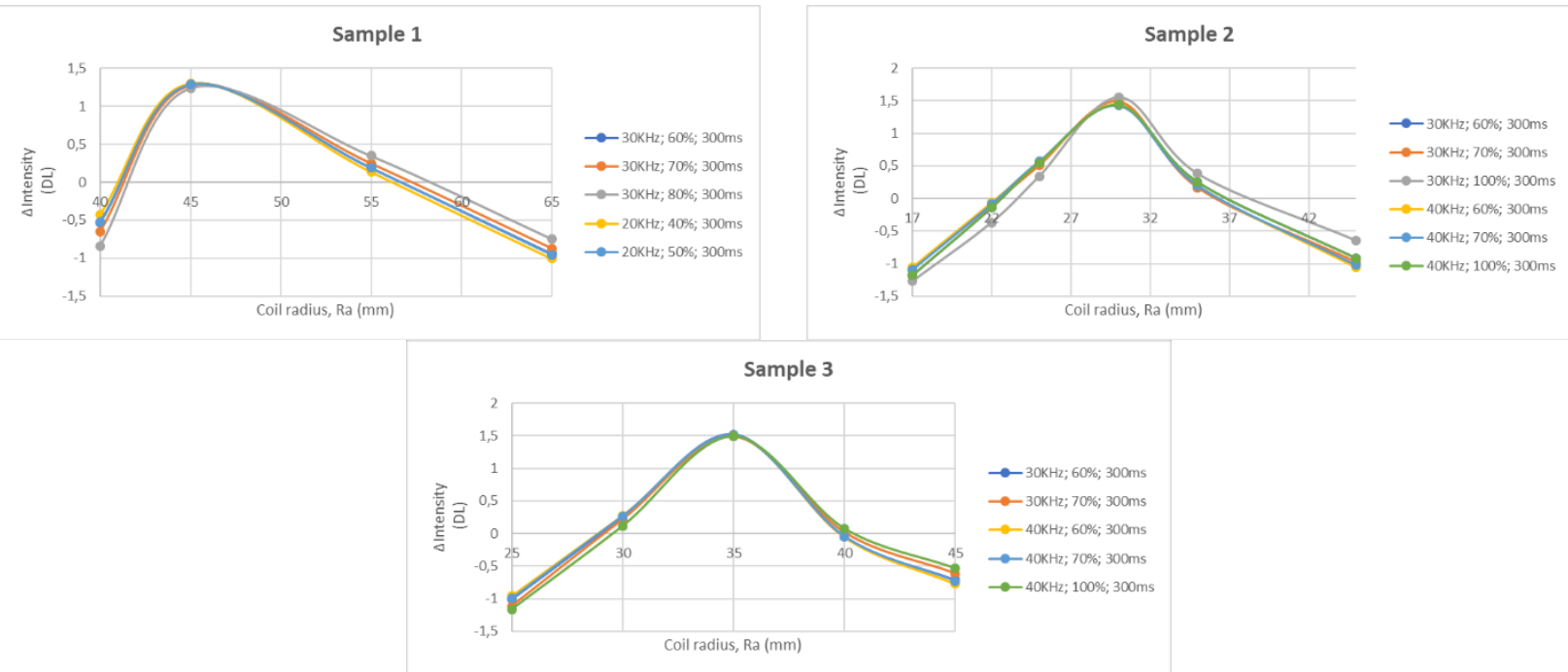

Table 6 shows, for each sample, the final graphs (average value at each point after normalization of data) for the different set of induction parameters employed.

As can be seen in Table 6, there is a common pattern between the three samples. The maximum for $\Delta$ Intensity is given for coil radiuses higher than sample radius. The shape of the curves is analogue for Sample 2 and 3 , but differs for Sample 1. In the graph of Sample 1, can be appreciated that there is a big gap between 45 and $55 \mathrm{~mm}$ where no coil has been manufactured. An extra coil of between those two values is needed in order to check if the position of the maximum is altered.

Regarding the ratio between "optimal" coil radius and sample radius, in Table 7 can be seen that increasing the radius of the sample leads to lower ratio values.

Table 7. Ratio between sample radius and optimal coil radius.

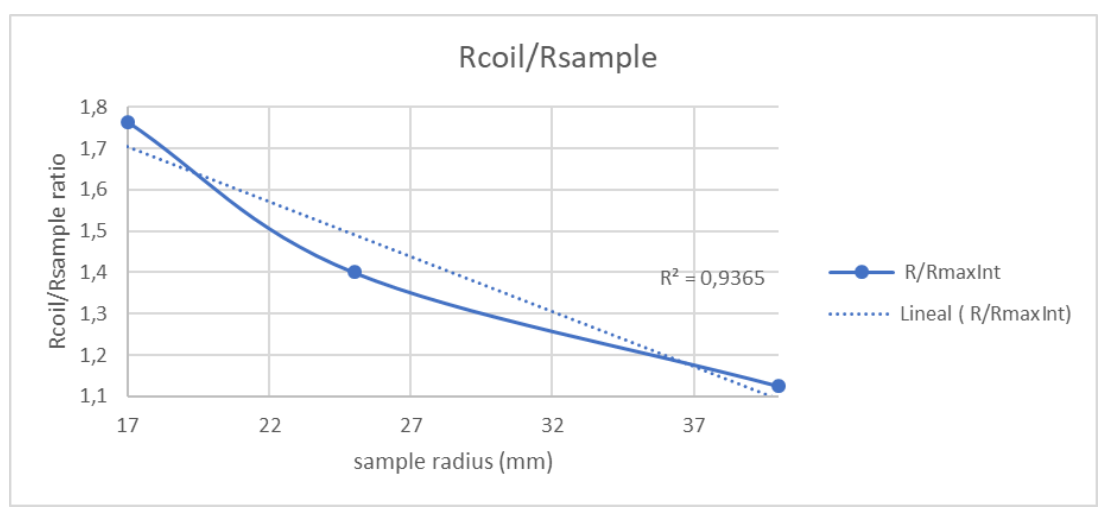

Due to the large size and mass of the links, it is necessary to ensure heating in order to carry out the inspections by inductive thermography. For this reason, the same study was carried out in the case of Sample 1 with a $5 \mathrm{~kW}$ generator. The intention is to verify that the heating increases and the conclusions of optimal radius are maintained at higher powers.

Table 8 shows the graphs obtained after processing the data, comparing $3 \mathrm{~kW}$ with $5 \mathrm{~kW}$. Only one set of induction parameters has been chosen to make the comparison.

- Induction Frequency: $30 \mathrm{kHz}$

- Power percentage: $60 \%$

- $\quad$ Pulse Length: $300 \mathrm{~ms}$ 


\subsection{1/qirt.2020.024}

$15^{\text {th }}$ Quantitative InfraRed Thermography Conference, 6 - 10 July 2020, Porto, Portugal

Table 8. Comparison between data obtained using $3 \mathrm{~kW}$ and $5 \mathrm{~kW}$ as excitation power. Sample 1; $30 \mathrm{kHz} ; 60 \% ; 300 \mathrm{~ms}$.

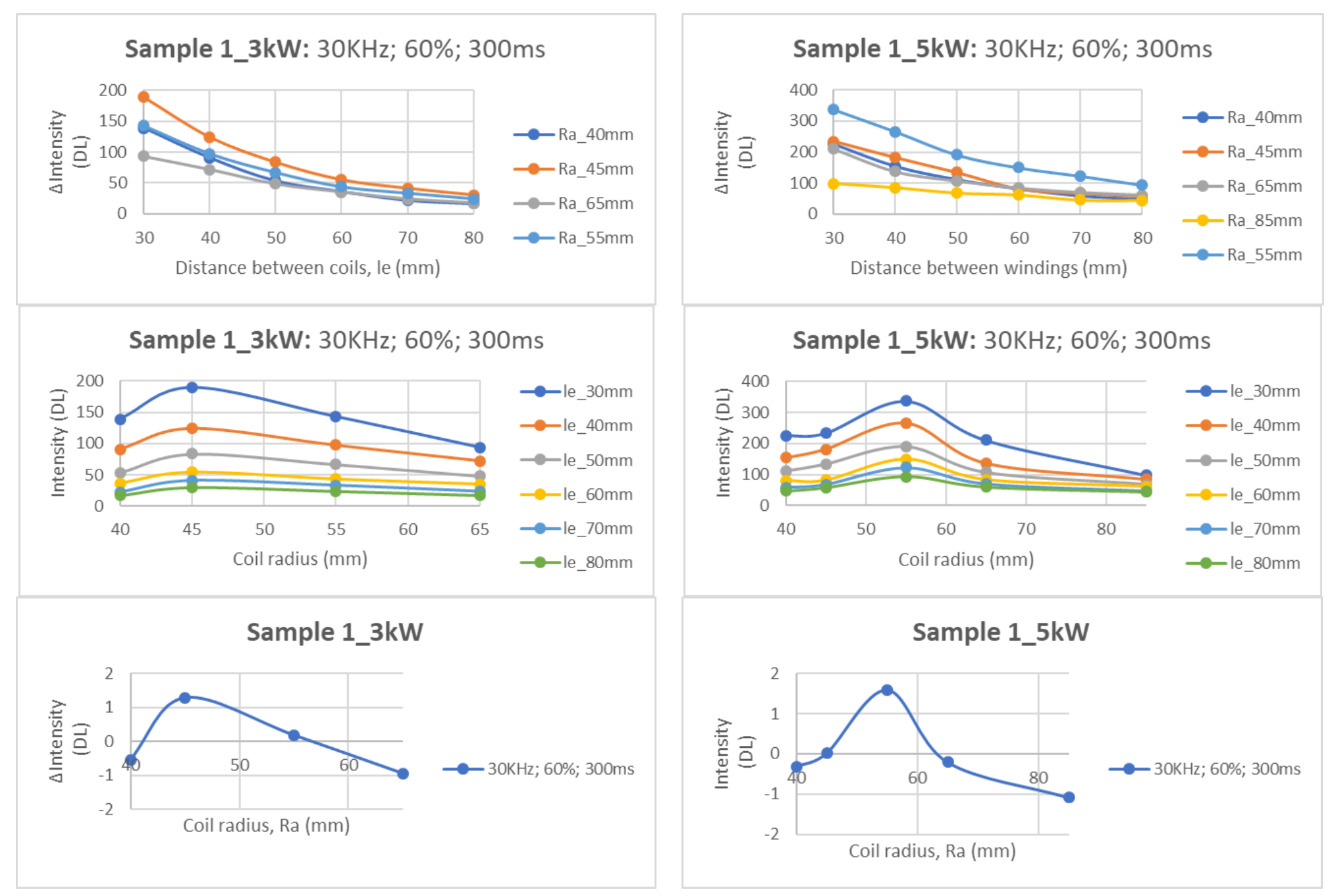




\section{Conclusions and Ongoing work}

\subsection{Experimental results: conclusions}

- $\quad$ There is a Helmholtz coil radius, larger than the sample radius, which maximizes the intensity increase.

- This conclusion is independent of the radius of the sample inspected and its material.

- $\quad$ Both Sample 2 and Sample 3 have a similar graph form. Sample 1 differs a little, and may be due to the lack of intermediate coil radius between those used.

- As the distance between the windings increases, the intensity increase is lower.

- By increasing the power of the induction generator, the existence of an "ideal" inspection radius is maintained, although its value shifts to somewhat higher values.

\subsection{Comparison between analytical model and experimental results}

- In agreement with the analytical model, the experimental tests have shown that the increase of the distance between windings reduces the heating performance.

- According to the analytical model, the optimal coil radius should be obtained when it is fitted to the chain link. However, experimental results have shown that the optimal coil radius is achieved with a bigger radius (Table 7).

The reason why the magnetic losses are maximum with a bigger coil radius is related to the equivalent electric circuit of the system (Fig. 9). As it is well known, the optimal working point in induction heating systems is achieved when the system works in resonance, increasing the feed current by means of the cancelation of both voltages, the inductive and capacitive. In our power electronic, the capacitance cannot be modified. Therefore, the resonance point is defined by the frequency and equivalent inductance, resulting in a resonance for this case when the distance between inductor and DUT is a little bit bigger. However, according to the developed analytical model, it can be deduced that the system could be improved reducing the coil radius and hence increasing the capacitance due to the decrease of the equivalent inductance.

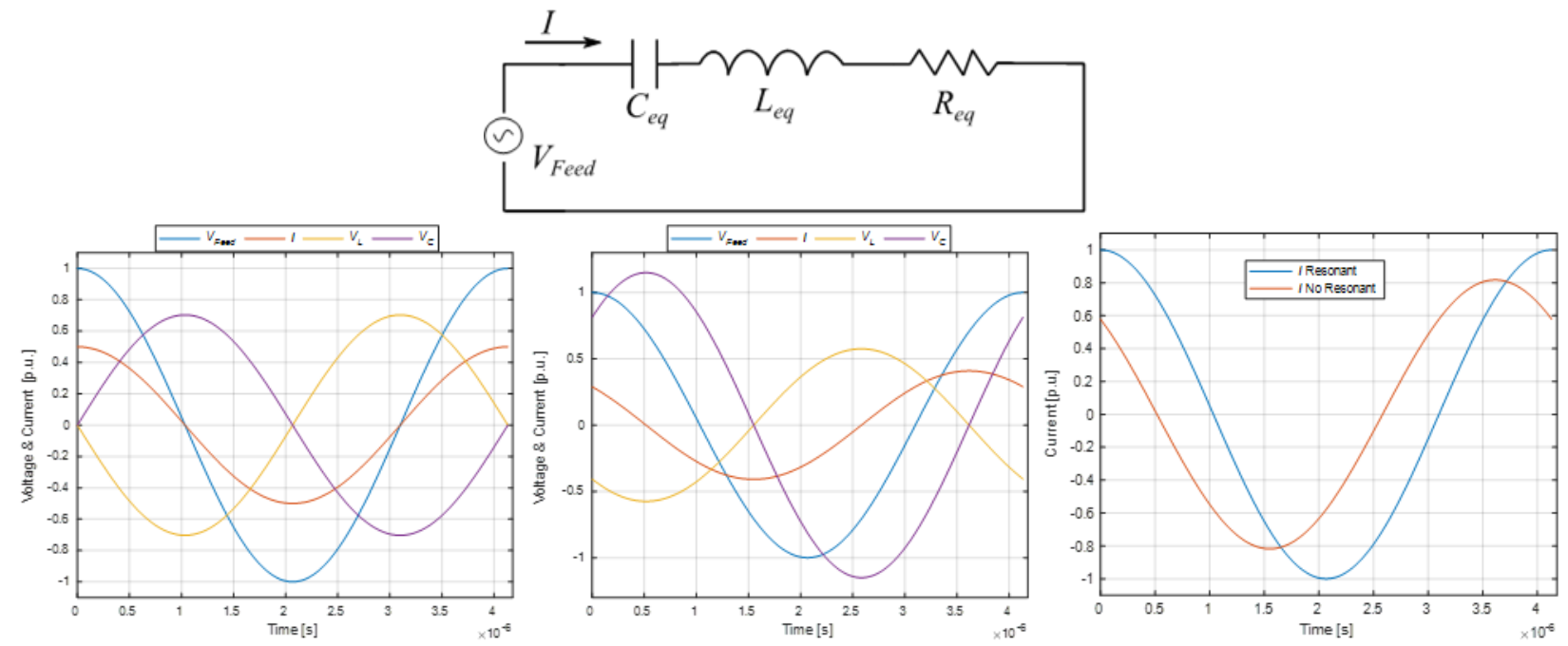

Fig. 9. Analysis of the electrical parameters.

\subsection{Ongoing work}

As it has been explained above, the present inductor generator does not fit the capacitance value to the equivalent circuit inductance to work in resonance. Therefore, the heating performances of the system are reduced. That is the reason why, it is pointed as future work the development of a platform that adjust the capacitance value depending on the system inductance. 
Besides this, in order to give one step more to the industrial inspection of coil link chains, automated inspections have been performed to stablish the feasibility of the procedure in a real situation. Different inductor geometries are defined to compare the detection results (Fig. 10).
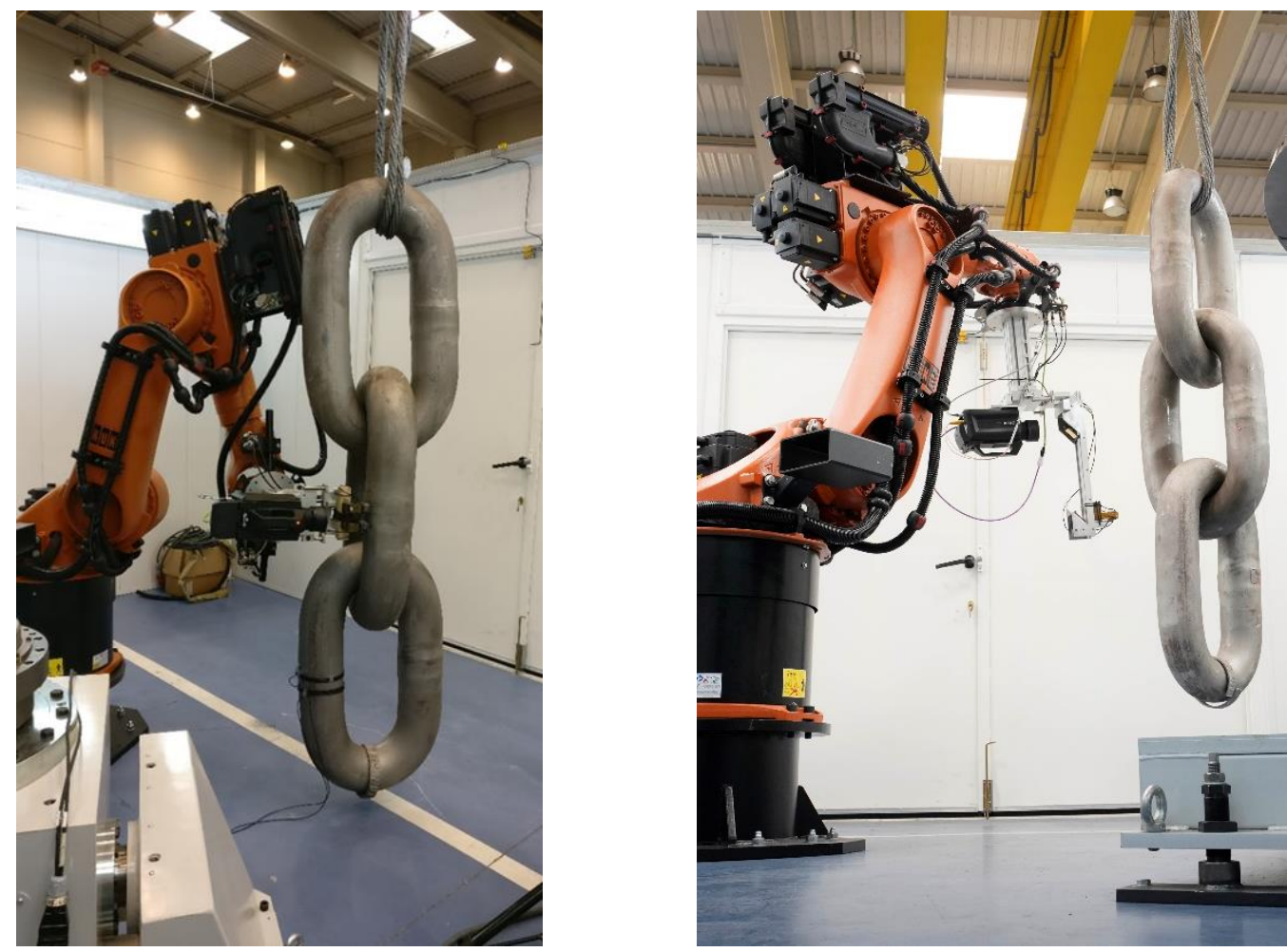

Fig. 10. Automated inspection of coil link chains at Lortek.

\section{References}

[1] C. Srajbr, A. Dillenz and K. Bräutigam, "Crack Detection at Aluminum Fuselages by Induction Excitec Thermography," 4th International Symposium on NDT in Aerospace 2012 - Th.3.A.3, 2012.

[2] C. Srajbr, "Induction excited thermography in industrial applications," 19th World Conference on Non Destructive Testing 2016, 2016.

[3] U. Netzelmann, G. Walle, S. Lugin, A. Ehlen, S. Bessert and B. Valeske, "Induction thermography: principle applications and first steps towards standardisation," Quantitative InfraRed Thermography Journal, 2016.

[4] J. Peng, G. Y. Tian, L. Wang, Y. Zhang, K. Li and X. Gao, "Investigation into eddy current pulsed thermograph) for rolling contact fatigue detection and characterization," NDT\&E International, 2015.

[5] R. Usamentiaga, P. Venegas, J. Guerediaga, L. Vega, F. Molleda and F. G. Bulnes, "Infrared Thermography fo| Temperature Measurement and Non-Destructive Testing," Sensors 2014, 14, 12305-12348, 2014.

[6] B. Oswald-Tranta, "Thermo-inductive crack detection," Nondestructive Testing and Evaluation, 22:2-3, 2007.

[7] G. Bertotti, "General properties of power losses in soft ferromagnetic materials," IEEE Transactions or Magnetics, vol. 24, pp. 621-630, 1988.

[8] C. P. Steinmetz, "On the Law of Hysteresis," Trans. Am. Inst. Electr. Eng. , vol. IX, no. 1, pp. 1-64, 1892.

[9] C. Steinmetz, "On the Law of Hysteresis," Proc. IEEE, vol. 72, no. 2, pp. 197-221, 1984.

[10] S. Zinn and S. L. Semiatin, Elements of Induction heating: Design, Control and Applications., California: ASN International, 1988. 